\title{
A FUNCTIONAL CALCULUS USING SINGULAR LAPLACE INTEGRALS
}

\author{
BY \\ EDWARD NELSON
}

1. Introduction. The problem of defining an operator $f(A)$ when $f$ is a function of a complex variable and $A$ is an operator on a Banach space, and studying its dependence on $f$ and $A$, has been approached in many different ways. E. Hille [8] has developed such a functional calculus by using Laplace integrals. The central idea is this: First one solves the problem for the functions of $\lambda$ given by $e^{\lambda t}, t \geqq 0$. That is, a meaning is attached to $P^{t}=e^{A t}$ for a class of operators $A$. Let $f$ be a function which is representable as $f(\lambda)=F\left(e^{\lambda t}\right)$, where $F$ is some linear functional defined on a domain of functions of $t, t \geqq 0$. Then if a meaning can be attached to $F\left(P^{t}\right)$ this is taken as the definition of $f(A)$. In the case studied by Hille, $F$ is a finite measure. Here we shall study the situation when $F$ is a finite combination of measures and differentiations-a distribution in the sense of Laurent Schwartz [11](1).

The larger class of functions obtained in this way includes the square root function. An application is made to the problem of square roots in Banach algebras. We also study from the point of view of this functional calculus the semigroups $Q^{t}$ which R. S. Phillips [10] obtained from a given semigroup $P^{t}$ by integrating with respect to a convolution semigroup of probability measures on $[0, \infty)$. If the transformations $P^{t}$ are associated with a Markoff process then the $Q^{t}$ are associated with another Markoff process which is subordinate to the original process in the sense of Bochner [3]. We obtain a characterization of the sample functions of the subordinate process in terms of the original process. This is exemplified in the case of the Markoff processes associated with the stable distribution functions (cf. [2] and [6]), particularly the Cauchy process, which are subordinate to the Brownian motion process.

2. A space of functionals and their Laplace transforms. Let $p$ be a positive measurable function of $t, 0 \leqq t<\infty$, such that $p(t+s) \leqq p(t) p(s)$. In the applications, $p$ will be of the form $p(t)=\|P t\|$ for some semigroup of operators $P^{t}$. We shall assume that $\omega_{0}$ is such that for each $\omega>\omega_{0}$ there is an $M$ for which

$$
p(t) \leqq M e^{\omega t}, \quad 0 \leqq t<\infty .
$$

Let $C^{n}(p)$ be the vector space of functions $\phi$ on $[0, \infty)$ with $n$ continuous

Received by the editors October 19, 1956.

(1) The referee has informed the author that a similarfunctional calculus has been developed by Schwartz in mimeographed notes and by Balakrishnan [1]. 
derivatives on $[0, \infty)$ such that $\left|\phi^{(i)}(t)\right| \leqq C p(t)$ for some $C$ and $i=0, \cdots, n$. Let $\mathfrak{F}^{n}(p)$ be the space of all linear functionals $F$ on $C^{n}(p)$ of the form

$$
F=\sum_{i=0}^{n} \mu_{i} \frac{d^{i}}{d t^{i}}
$$

where the $\mu_{i}$ are complex measures such that $\int_{0}^{\infty} p(t) d\left|\mu_{i}\right|(t)<\infty, i=0, \cdots, n$. That is

$$
F(\phi)=\sum_{i=0}^{n} \int_{0}^{\infty} \phi^{(i)}(t) d \mu_{i}(t) .
$$

Notice that the $\mu_{i}$ need not be uniquely determined. If $\int_{0}^{\infty} p(t) d|\mu|(t)<\infty$, then the functional $\mu(\phi)=\int_{0}^{\infty} \phi(t) d \mu(t)$ is in $\mathfrak{F}^{0}(p)$. The spaces $\mathfrak{F}^{n}(p)$ form an increasing sequence. We denote their union by $\mathfrak{F}(p)$.

A special case is that in which $p(t)=1,0 \leqq t<\infty$. In the terminology of L. Schwartz [11, Chapter VI], $\mathfrak{F}(1)$ is the subset of the space of distributions in $\mathfrak{D}_{L^{1}}^{\prime}$ having support on the right half axis. This follows from [11, Chapter VI, Theorem XXV]. However, this fact will not be used in the following.

If $F$ is in $\mathfrak{F}^{n}(p)$ and $G$ is in $\mathfrak{F}^{m}(p)$ then $H$ defined by

$$
H(\phi)=F_{s}\left(G_{t}(\phi(t+s))\right), \quad \phi \in C^{n+m}(p)
$$

(where a subscript on the symbol for a functional indicates that the integration in (3) is with respect to that variable) is readily seen to be in $\mathfrak{F}^{n+m}(p)$. In fact, if $F=\sum_{i=0}^{n} \mu_{i}\left(d^{i} / d t^{i}\right), G=\sum_{j=0}^{m} \nu_{j}\left(d^{j} / d t^{j}\right)$, then it is easily verified that $H=\sum_{k=0}^{n+m} \rho_{k}\left(d^{k} / d t^{k}\right)$, where $\rho_{k}=\sum_{i+j=k} \mu_{i} * \nu_{j}$. Here $\mu * \nu$, the convolution of $\mu$ and $\nu$, denotes the measure such that

$$
\int_{0}^{\infty} f(u) d \mu * \nu(u)=\int_{0}^{\infty} \int_{0}^{\infty} f(t+s) d \mu(t) d \nu(s) .
$$

Then

$$
\begin{aligned}
\int_{0}^{\infty} p(u) d\left|\rho_{k}\right|(u) & \leqq \sum_{i+j=k} \int_{0}^{\infty} \int_{0}^{\infty} p(t+s) d\left|\mu_{i}\right|(t) d\left|\nu_{j}\right|(s) \\
& \leqq \sum_{i+j=k} \int_{0}^{\infty} \int_{0}^{\infty} p(t) p(s) d\left|\mu_{i}\right|(t) d\left|\nu_{j}\right|(s)<\infty .
\end{aligned}
$$

The functional $H$ is called the convolution of $F$ and $G, H=F * G$. Thus $\mathfrak{F}(p)$ is closed under convolution.

If $F$ is in $\mathfrak{F}(p)$ we call

$$
\tilde{F}(\lambda)=F_{t}\left(e^{\lambda t}\right), \quad \operatorname{Re}(\lambda)<\omega_{0}
$$

the Laplace transform of $F$. The definition (5) will be more convenient than 
the usual one in which $\lambda$ is replaced by $-\lambda$. By (1) and (2), $F$ is holomorphic in $\operatorname{Re}(\lambda)<\omega_{0}$.

The class of Laplace transforms of functionals in $\mathfrak{F}(p)$ will be called $\tilde{F}(p)$. Since $\mathfrak{F}(p)$ is closed under convolution, $\widetilde{F}(p)$ is closed under multiplication, since

$$
(F * G)^{\sim}(\lambda)=F_{s}\left(G_{t}\left(e^{\lambda(t+s)}\right)\right)=F_{s}\left(e^{\lambda s}\left(G_{t}\left(e^{\lambda t}\right)\right)=F_{s}\left(e^{\lambda s}\right) G_{t}\left(e^{\lambda t}\right)=\tilde{F}(\lambda) \tilde{G}(\lambda) .\right.
$$

Notice also that by (2),

$$
\widetilde{F}(\lambda)=\sum_{i=0}^{n} \int_{0}^{\infty} \lambda^{i} e^{\lambda t} d \mu_{i}(t)=\sum_{i=0}^{n} \lambda^{i} \tilde{\mu}_{i}(\lambda) .
$$

Therefore $\tilde{F}(p)$ is the algebra generated by the polynomials and the Laplace transforms of measures in $\mathfrak{F}^{0}(p)$.

Let $\nu_{j}$ be a sequence of positive measures on $[0, \infty)$ such that $d \nu_{j}(s)$ $=\psi_{j}(s) d s$ where the $\psi_{j}$ have $n$ continuous derivatives on $(-\infty, \infty)$ (so that $\left.\psi_{j !}^{(i)}(0)=0, i=0, \cdots, n\right), \nu_{j}[0,1 / j]=\nu_{j}[0, \infty)=1$. Let $F=\sum_{i=0}^{n} \mu_{i}\left(d^{i} / d t^{i}\right)$ be in $\mathfrak{F}^{(n)}(p)$. Then for all $\phi$ in $C^{n}(p)$,

$$
\begin{aligned}
F * \nu_{j}(\phi) & =\sum_{i=0}^{n} \int_{0}^{\infty} \int_{0}^{\infty} \phi^{(i)}(t+s) d \mu_{i}(t) \psi_{j}(s) d s \\
& =\sum_{i=0}^{n}(-1)^{i} \int_{0}^{\infty} \int_{0}^{\infty} \phi(t+s) d \mu_{i}(t) \psi_{j}^{(i)}(s) d s
\end{aligned}
$$

on integration by parts. Thus $F * \nu_{j}$ is in $\mathfrak{F}^{0}(p)$. By the well-known uniqueness theorem for the Laplace transform of measures (cf. [8]), $F * \nu_{j}=0$ if $\left(F * \nu_{j}\right) \sim(\lambda)$ $=\tilde{F}(\lambda) \tilde{\nu}_{j}(\lambda)=0, \operatorname{Re}(\lambda)<\omega_{0}$, and so $F * \nu_{j}=0$ if $\widetilde{F}=0$. Now $\lim _{j} F * \nu_{j}(\phi)=F(\phi)$ for $\phi$ in $C^{n}(p)$, since $\nu_{j}$ is a probability measure with support contained in $[0,1 / j]$. Therefore, if $\tilde{F}=0$, then $F * \nu_{j}=0$ and so $F=0$. Thus for all $F$ in $\mathfrak{F}(p), F=0$ if and only if $\widetilde{F}=0$.

3. Strongly continuous semigroups and their infinitesimal generators. By a strongly continuous semigroup we shall mean a family of bounded operators $P^{t}, 0 \leqq t<\infty$, on a Banach space $\mathfrak{X}$ such that $P^{t} P^{s}=P^{t+s}, P^{0}=1$, and $P^{t} x \rightarrow x$ as $t \rightarrow 0$ for all $x$ in $\mathfrak{X}$. The infinitesimal generator $A$ of $P^{t}$ is the operator defined by

$$
A x=\lim _{t \rightarrow 0} t^{-1}\left(P^{t} x-x\right)
$$

on the domain $D(A)$ of all $x$ for which the limit exists. We recall the following facts from the theory of semigroups (proofs are in [8]):

The operator $A$ is closed and densely defined. The limit

$$
\omega_{0}=\lim _{t \rightarrow \infty} t^{-1} \log \left\|P^{t}\right\|
$$


exists, and for any $\omega>\omega_{0},\left\|P^{t}\right\| \leqq M e^{\omega t}$ for some $M$. If $\operatorname{Re}(\lambda)>\omega_{0}$, then the resolvent $R(\lambda ; A)=(\lambda-A)^{-1}$ exists and

$$
R(\lambda ; A) x=\int_{0}^{\infty} e^{-\lambda t} P^{t} x d t, \quad x \in \mathfrak{X} .
$$

(The integral in (8) and all strong integrals appearing in this paper are Bochner integrals, as in [8].) The domain $D\left(A^{n}\right)$ of $A^{n}$ is dense for all $n$ and

$$
\frac{d^{n}}{d t^{n}} P^{t} x=P^{t} A^{n} x=A^{n} P^{t} x, \quad x \in D\left(A^{n}\right) .
$$

4. The functional calculus. Let $A$ be the infinitesimal generator of the contraction semigroup $P^{t}$, let $p(t)=\left\|P^{t}\right\|$, let $f$ be the Laplace transform of a functional $F$ in $\mathfrak{F}^{n}(p)$ but not $\mathfrak{F}^{n-1}(p)$, and let the $\mu_{i}, i=0, \cdots, n$, be the measures in a representation (2) of $F$. Let

$$
f(A) x=\sum_{i=0}^{n} \int_{0}^{\infty} P^{t} A^{i} x d \mu_{i}(t), \quad x \in D\left(A^{n}\right) .
$$

This is independent of the choice of the $\mu_{i}$, since for all $y$ in the dual space $\mathfrak{X}^{*}$ we have by (9) that

$$
(f(A) x, y)=F_{t}\left(P^{t} x, y\right)
$$

so that $f(A) x, x \in D\left(A^{n}\right)$, depends only on $F$. Since we showed at the end of $\$ 2$ that $F$ is uniquely determined by its Laplace transform $f, f(A) x$ is well-defined for all $x \in D\left(A^{n}\right)$. We want to show that $f(A)$ on $D\left(A^{n}\right)$ has a closure.

Lemma 1. If $A$ is an operator on a Banach space $\mathfrak{X}$ with a nonempty resolvent set and if the $B_{i}, i=0, \cdots, n$ are bounded operators, then the operator $C=\sum_{i=0}^{n} A^{i} B_{i}$ has a closure.

Proof. Let $B_{j}^{\prime}=\sum_{i=j}^{n} C_{i, j} \lambda^{i-i} B_{i}$. Let $C^{\prime}=\sum_{j=0}^{n}(A-\lambda)^{i} B_{j}^{\prime}$. Then if $x$ is in the domain of $C$, it follows that for $i \geqq j, B_{i} x \in D\left(A^{i}\right) \subset D\left(A^{j}\right)$ and $B_{j}^{\prime} x$ $\in D\left((A-\lambda)^{i}\right)$, so that $x$ is in the domain of $C^{\prime}$. By the binomial theorem, $C^{\prime} x=C x$. Thus $C^{\prime} \supset C$. (It is easily seen that $C^{\prime}=C$, but we do not need this.)

Now choose $\lambda$ in the resolvent set of $A$, so that $(A-\lambda)^{-1}$ is a bounded operator defined on all of $\mathfrak{X}$. Let $x_{j}$ be in the domain of $C$ with $x_{j} \rightarrow 0, C x_{j} \rightarrow x$. We wish to show that $x=0$. Since $C^{\prime} x_{j} \rightarrow x$ and $(A-\lambda)^{-n}$ is bounded, $(A-\lambda)^{-n} C^{\prime} x_{j} \rightarrow(A-\lambda)^{-n} x$. But $(A-\lambda)^{-n} C^{\prime}=\sum_{j=0}^{n}(A-\lambda)^{-n+j} B_{j}^{\prime}$ is also bounded. Since $x_{j} \rightarrow 0, \quad(A-\lambda)^{-n} C^{\prime} x_{j} \rightarrow 0$, and $0=(A-\lambda)^{-n} x$. Therefore $0=(A-\lambda)^{n}(A-\lambda)^{-n} x=x$. It follows by linearity that if $x_{j} \rightarrow y, x_{j}^{\prime} \rightarrow y, C x_{j} \rightarrow z$, $C x_{j}^{\prime} \rightarrow z^{\prime}$ then $z=z^{\prime}$, so that we may define $\bar{C} y=z$ whenever $x_{j} \rightarrow y$ and $C x_{j} \rightarrow z$, obtaining a well-defined closed extension $\bar{C}$ of $C$. 
In the case under consideration, we may define $B_{i} x=\int_{0}^{\infty} P^{t} x d \mu_{i}(t)$ so that by (10) and (9), $f(A)=\sum_{i=0}^{n} B_{i} A^{i}=\sum_{i=0}^{n} A^{i} B_{i}$ on $D\left(A^{n}\right)$. Thus $f(A)$ on $D\left(A^{n}\right)$ has a closure. We shall denote this closure $f(A)$, and its domain $D(f(A))$.

Lemma 2. Let $f=\widetilde{F}, F$ in $\mathfrak{F}^{n}(p)$ but not $\mathfrak{F}^{n-1}(p)$. Then for any $k \geqq n, f(A)$ is the closure of its restriction to $D\left(A^{k}\right)$. If $W_{j}$ is defined by

$$
W_{j} x=j \int_{0}^{1 / j} P^{t} x d t, \quad x \in \mathfrak{X},
$$

then $x$ is in the domain of $f(A)$ if and only if $\lim _{j} f(A) W_{j}^{k} x$ exists.

Proof. $s^{-1}\left(P^{8} W_{j} x-W_{j} x\right)=-j s^{-1} \int_{0}^{s} P^{t} x d t+j s^{-1} \int_{1 / j}^{1 / j+s} P^{t} x d t$, and as $s \rightarrow 0$ this converges to $-j x+j P^{1 / i} x$. Thus $W_{j} x \in D(A)$ for all $x$ in $\mathfrak{X}$. Also, $W_{j} x \rightarrow x$ for all $x$ in $\mathfrak{X} .\left\|W_{j}\right\| \leqq M e^{\omega}$ is uniformly bounded, so that $W_{j}^{k} x \rightarrow x$ for all $x \in \mathfrak{X}$, and $W_{j}^{\boldsymbol{k}} x$ is in $D\left(A^{k}\right)$.

To prove the second statement of the lemma, let $f(A) W_{j}^{k} x \rightarrow y$. Since $W_{j}^{k} x \rightarrow x$ and $f(A)$ is closed, $x$ is in $D(f(A)), f(A) x=y$. Conversely, suppose that $f(A) x=y$. Then there is a sequence $x_{m}$ in $D\left(A^{n}\right)$ such that $x_{m} \rightarrow x$, $f(A) x_{m} \rightarrow y$, by definition of $f(A)$. For fixed $j$ we have by (10) that $f(A) W_{j}^{k} x$ $=\lim _{m} f(A) W_{j}^{k} x_{m}=\lim _{m} W_{j}^{k} f(A) x_{m}=W_{j}^{k} f(A) x$. Therefore $\lim _{j} f(A) W_{j}^{k} x$ $=\lim _{j} W_{j}^{k} f(A) x=f(A) x$.

The first statement of the lemma, that $f(A)$ is the closure of its restriction to $D\left(A^{k}\right)$, follows from this, since $W_{j}^{k} x$ is in $D\left(A^{k}\right)$. This proves Lemma 2.

Lemma 3. If $f$ and $g$ are in $\mathfrak{F}(p)$ then $(f+g)(A)$ is the closure of $f(A)+g(A)$ and $(f g)(A)$ is the closure of $f(A) g(A)$.

Proof. Let $f=\tilde{F}$ be in $\tilde{F}^{m}(p), g=\tilde{G}$ be in $\tilde{\mathfrak{F}}^{n}(p)$, and let $k=m+n$. Let $x$ be in the domain $D(f(A)) \cap D(g(A))$ of $f(A)+g(A)$. By Lemma 2, $(f(A)+g(A)) x$ $=\lim _{j}\left(f(A) W_{j}^{k} x+g(A) W_{j}^{k} x\right)=\lim _{j}(f+g)(A) W_{j}^{k} x=(f+g)(A) x$, so that $f(A)$ $+g(A) \subset(f+g)(A)$. Since the domain of $f(A)+g(A)$ includes $D\left(A^{k}\right),(f+g)(A)$ is the closure of $f(A)+g(A)$ by Lemma 2 .

Let $x$ be in $D\left(A^{k}\right)$. Then for all $y$ in $\mathfrak{X}^{*},((f g)(A) x, y)=(F * G)_{u}\left(P^{u} x, y\right)$ $=F_{s}\left(G_{t}\left(P^{t+s} x, y\right)\right)=F_{s}\left(g(A) P^{s} x, y\right)=F_{s}\left(P^{s} g(A) x, y\right)=(f(A) g(A) x, y)$, so that $(f g)(A) x=f(A) g(A) x$. Now let $x$ be an arbitrary element in the domain of $f(A) g(A)$. Then $f(A) g(A) x=\lim _{j} f(A) g(A) W_{j}^{k} x=\lim _{j}(f g)(A) W_{j}^{k} x=(f g)(A) x$, so that $f(A) g(A) \subset(f g)(A)$. By Lemma $2,(f g)(A)$ is the closure of $f(A) g(A)$.

The closure of the sum (product) of two closed operators is called the strong sum (product). Thus Lemma 3 states that the map $f \rightarrow f(A)$ is a homomorphism, provided the algebraic operations on the $f(A)$ are understood in the strong sense. The following theorem summarizes the results of this section.

TheOREm 1. If $A$ is the infinitesimal generator of a strongly continuous 
semigroup $P^{t}, p(t)=\left\|P^{t}\right\|$, then there is a homomorphism $f \rightarrow f(A)$ from the algebra $\widetilde{F}(p)$ generated by the polynomials and the Laplace transforms of measures $\mu$ such that $\int_{0}^{\infty} p(t) d|\mu|(t)<\infty$ to an algebra of closed, densely defined operators, the algebraic operations being understood in the sense of strong sums and products. If $f$ is the Laplace transform of a functional $F$ given by (2) then $D\left(A^{n}\right)$ is included in the domain of $f(A), f(A)$ is the closure of its restriction to $D\left(A^{n}\right)$, and $f(A) x$ is given by (10) for all $x$ in $D\left(A^{n}\right)$.

N.B. It should be remembered that with our definition $\widetilde{F}(\lambda)=F_{t}\left(e^{\lambda t}\right)$ of Laplace transform the functions in $\mathfrak{F}(p)$ are holomorphic in some left half plane.

CoRollary. If $A$ is a bounded operator or if $f$ is the Laplace transform of a measure $\mu$ in $F^{0}(p)$, then $f(A)$ is a bounded operator.

Proof. This follows from (10).

5. Fractional differentiation and powers of $\lambda$. The operation of taking the fractional derivative of order $\alpha$ is a functional of the type we are considering (see Schwartz [11]). This has Laplace transform $\lambda^{\alpha}$ so that under appropriate restrictions on $\left\|P^{t}\right\|$ our functional calculus enables us to form $A^{\alpha}$.

Theorem 2. Let $1>\operatorname{Re}(\alpha)>0$. If $\int_{1}^{\infty}\left(p(t) / t^{\alpha+1} d t\right)<\infty$ then $\lambda^{\alpha} \in \tilde{F}(p)$. If $F(\phi)=-\left(1 / \Gamma^{(-\alpha)}\right) \int_{0}^{\infty}\left((\phi(t)-\phi(0)) / t^{\alpha+1}\right) d t$ then $F \in \mathfrak{F}^{1}(p)$ and $\widetilde{F}(\lambda)=-(-\lambda)^{\alpha}$.

Proof. If $\phi \in C^{1}(p)$, then integration by parts shows that

$$
-\Gamma(-\alpha) F(\phi)=\phi(1)-\phi(0)+\frac{1}{\alpha} \int_{0}^{1} \frac{\phi^{\prime}(t)}{t^{\alpha}} d t+\int_{1}^{\infty} \frac{\phi(t)-\phi(0)}{t^{\alpha+1}} d t .
$$

Here we have used the facts that $t^{-\alpha}(\phi(t)-\phi(0)) \rightarrow 0$ as $t \rightarrow 0$, since $\phi$ has a continuous first derivative on $[0, \infty)$ and $1>\operatorname{Re}(\alpha)$, and that the last integral on the right converges absolutely since $|\phi(t)| \leqq C p(t)$. But (12) is of the form (3) with $n=1$, and so $F \in \mathscr{F}^{1}(p)$. To calculate the Laplace transform $\tilde{F}(\lambda)$ $=-(1 / \Gamma(-\alpha)) \int_{0}^{\infty}\left(\left(e^{\lambda t}-1\right) / t^{\alpha+1}\right) d t$ we may make the substitution $u=-\lambda t$ and integrate by parts, yielding $\tilde{F}(\lambda)=-(-\lambda)^{\alpha}$. Thus $\tilde{F}(\lambda)$, and hence $\lambda^{\alpha}$ $=-(-1)^{-\alpha} \widetilde{F}(\lambda)$, is in $\widetilde{F}(p)$.

6. Square roots in Banach algebras. Let $\mathfrak{A}$ be a Banach algebra with unit, $x$ an element of $\mathfrak{A}$ with spectrum contained in the unit circle. If the spectrum of $x$ is contained in the interior of the unit circle, it is shown by means of Cauchy's integral that $1-x$ has a square root in $\mathfrak{A}$ (see [9]). If $\|x\| \leqq 1$, the power series $\sum_{n=0}^{\infty}(-1)^{n} C_{1 / 2, n} x^{n}$ converges absolutely, and so $1-x$ has a square root in $\mathfrak{A}$. By using Laplace integrals we may prove a slightly stronger result.

Theorem 3. If $\mathfrak{A}$ is a Banach algebra with unit, $x$ is in $\mathfrak{A}$, and

$$
\sum_{n=0}^{\infty} \frac{\left\|x^{n}\right\|}{n^{3 / 2}}<\infty
$$


then $1-x$ has a square root $x_{0}$ in $\mathfrak{A}$, given by the absolutely convergent integral

$$
x_{0}=-\frac{1}{2 \pi^{1 / 2}} \int_{0}^{\infty} \frac{e^{-t(1-x)}-1}{t^{3 / 2}} d t .
$$

Proof. Let $P^{t} y=e^{-t(1-x)} y$ for all $y$ in $\mathfrak{A}$. Then $P^{t}$ is a strongly continuous (in fact, uniformly continuous) semigroup whose infinitesimal generator is $A y=-(1-x) y$.

$$
\begin{aligned}
& \int_{1}^{\infty} \frac{\left\|P^{\prime}\right\|}{t^{3 / 2}} d t \leqq \int_{1}^{\infty} \sum_{n=0}^{\infty} \frac{\left\|x^{n}\right\|}{n !} e^{-t} t^{n-3 / 2} d t \\
& \leqq C+\sum_{n=1}^{\infty} \frac{\left\|x^{n}\right\|}{n !} \int_{0}^{\infty} e^{-t} t^{n-3 / 2} d t=\sum_{n=1}^{\infty}\left\|x^{n}\right\| \frac{\Gamma(n-1 / 2)}{\Gamma(n+1)} .
\end{aligned}
$$

But $\Gamma(n-1 / 2) / \Gamma(n+1) \sim e^{-n+1 / 2}(n-1 / 2)^{n-1} / e^{-n-1}(n+1)^{n+1 / 2}=O\left(n^{-3 / 2}\right)$ by Stirling's formula. Thus (14) is absolutely convergent if (13) holds. By Theorem 2, the operator $B y=x_{0} y$ is $f(A)$ where $f(\lambda)=(-\lambda)^{1 / 2}$. By the homomorphism property of the functional calculus (Theorem 1), $B^{2}=-A$, $x_{0}^{2} y=(1-x) y$ for all $y$ in $\mathfrak{R}, x_{0}^{2}=1-x$.

7. Convolution semigroups of probability measures on the half-axis. Let $\mathfrak{F}^{+}$be the subset of $\mathfrak{F}(1)$ consisting of all functionals of the form

$$
F(\phi)=\int_{0}^{\infty}(\phi(t)-\phi(0)) d m(t)+\gamma \phi^{\prime}(0)
$$

where $\gamma \geqq 0$ and $m$ is a positive measure such that $m(\epsilon, \infty)<\infty$ and $\int_{0}^{\epsilon} t d m(t)$ $<\infty$ for all $\epsilon>0$. As in $\$ 5$, this integral may be split into two and integration by parts applied to the first one, so that $F$ is in $\mathfrak{F}(1)$. Or we may appeal to Schwartz' theorem [11, Chapter VI] on the representation of distributions in $\mathscr{D}_{L^{1}}^{\prime}$, since $F$ clearly belongs to this space. The integral in (15) converges for $\phi$ in $C^{1}(1)$.

Let $\widetilde{\mathfrak{F}}^{+}$be the set of Laplace transforms of functionals in $\mathfrak{F}^{+}$. It follows from the characterization of infinitely divisible "distributions" (i.e. probability measures) by Lévy-Kolmogoroff-Khintchine-Feller (see Doob $[4$, p. 128]) that $f$ is in $\tilde{F}^{+}$if and only if $e^{t f}$ is the Laplace transform of a probability measure $\mu^{t}$ for each $t \geqq 0$. A proof of this statement is contained in a paper by R. S. Phillips [10]. The measures $\mu^{t}$ form a semigroup under convolution and $\mu^{t}(\epsilon, \infty) \rightarrow 0$ as $t \rightarrow 0$ for all $\epsilon>0$.

Let $P^{t}$ be a strongly continuous semigroup on a Banach space $\mathfrak{X}$ with infinitesimal generator $A$, such that $\left\|P^{t}\right\| \leqq 1$, and let

$$
Q^{t} x=\int_{0}^{\infty} P^{u} x d \mu^{t}(u), \quad x \in \mathfrak{X} .
$$

Then as R. S. Phillips shows in [10], $Q^{t}$ is a strongly continuous semigroup and its infinitesimal generator $B$ satisfies 


$$
B x=\int_{0}^{\infty}\left(P^{t} x-x\right) d m(t)+\gamma A x, \quad x \in D(A) .
$$

In fact, Phillips studies a more general case where the $\mu^{t}$ need not be finite or positive. In terms of the functional calculus, (17) may be written

$$
B x=f(A) x,
$$

where $f(\lambda)=\int_{0}^{\infty}\left(e^{t \lambda}-1\right) d m(t)+\gamma \lambda$.

The observation we wish to make is that $f(A)$ is equal to $B$. That is, $B$ is equal to the closure of its restriction to $D(A)$. To see this, let $\operatorname{Re}(\beta)>0$ and let $\nu$ be the measure given by $\nu(\phi)=\int_{0}^{\infty} e^{-\beta t} \mu^{t}(\phi) d t$. Then $\tilde{\nu}(\lambda)=\int_{0}^{\infty} e^{-\beta t} \tilde{\mu}^{t}(\lambda) d t$ $=\int_{0}^{\infty} e^{-\beta t} e^{t f(\lambda)} d t=1 /(\beta-f(\lambda))$. Thus $(\beta-f(A)) \tilde{\nu}(A) x=x$ for all $x$ in $\mathfrak{X}$ and $\tilde{\nu}(A)(\beta-f(A)) x=x$ for $x \in D(f(A))$. That is, $\tilde{\nu}(A)=R(f(A) ; \beta)$. Therefore $R(f(A) ; \beta)=\int_{0}^{\infty} e^{-\beta t} Q^{t} x d t, \operatorname{Re}(\beta)>0, x \in \mathfrak{X}$, and so $f(A)$ is the infinitesimal generator of $Q^{t}$.

Consider the function $-(-\lambda)^{\alpha}$ for $1>\operatorname{Re}(\alpha)>0$. By Theorem 2, these functions are in $\tilde{\mathfrak{F}}^{+}$. Therefore if $A$ is the infinitesimal generator of the strongly continuous semigroup $P^{t},\left\|P^{t}\right\| \leqq 1$, then $-(-A)^{\alpha}$, defined as the closure of $x \rightarrow-(1 / \Gamma(-\alpha)) \int_{0}^{\infty}\left(\left(P^{t} x-x\right) / t^{\alpha+1}\right) d t, x \in D(A)$ is the infinitesimal generator of a strongly continuous semigroup $Q^{t}$ related to $P^{t}$ by (16). The important consequence of the identification of the infinitesimal generator of $Q^{t}$ with the operator defined in this way is that the closure of $\left(-(-A)^{\alpha}\right)$ $\cdot\left(-(-A)^{\beta}\right)$ is $(-A)^{\alpha+\beta}$ by Theorem 1 . This property justifies the notation $-(-A)^{\alpha}$ for the infinitesimal generator of $Q^{t}$.

If $\mathfrak{X}$ is a partially ordered Banach space and $P^{t}$ is order preserving, then so is $Q^{t}$ given by (16). We shall examine this situation in more detail for the case of transformations associated with certain Markoff processes.

8. The sample functions of subordinate Markoff processes. We shall need a simple extension of the concept of the Cartesian product of two measure spaces. We will develop it only for probability spaces, although the construction is valid in more general cases.

We recall that a probability space $(\Omega, \mathcal{S}, \operatorname{Pr})$ is a set $\Omega$ with a class $\mathcal{S}$ of subsets, called measurable subsets, which is a Boolean algebra closed under the formation of countable unions and intersections, and a positive measure $\operatorname{Pr}$ such that $\operatorname{Pr}(\Omega)=1$.

Definition. Let $(\Omega, s, \operatorname{Pr})$ and $\left(\Omega^{\prime}, \mathcal{s}^{\prime}, \mathrm{Pr}^{\prime}\right)$ be two probability spaces. By their left product is meant the triple $\left(\Omega \times \Omega^{\prime}, \mathcal{S} \cdot \mathcal{S}^{\prime}, \operatorname{Pr} \cdot \operatorname{Pr}^{\prime}\right)$ where $\mathcal{S} \cdot \mathcal{S}^{\prime}$ consists of all subsets $C$ of $\Omega \times \Omega^{\prime}$ such that for each $\omega^{\prime} \in \Omega^{\prime}, C^{\omega^{\prime}}=\left\{\omega:\left(\omega, \omega^{\prime}\right) \in C\right\}$ is in $\delta$ and $\operatorname{Pr}\left(C^{\omega^{\prime}}\right)$ is a measurable function of $\omega^{\prime}$ (i.e., for each real Borel set $B$, $\left.\left\{\omega^{\prime}: \operatorname{Pr}\left(C^{\omega^{\prime}}\right) \in B\right\} \in \mathbb{s}^{\prime}\right)$, and

$$
\operatorname{Pr} \cdot \operatorname{Pr}^{\prime}(C)=\int_{\Omega^{\prime}} \operatorname{Pr}\left(C^{\omega^{\prime}}\right) d \operatorname{Pr}^{\prime}\left(\omega^{\prime}\right) .
$$


We will sometimes write $\Omega \cdot \Omega^{\prime}$ as an abbreviation for the triple $\left(\Omega \times \Omega^{\prime}\right.$, $\left.S \cdot \mathcal{S}^{\prime}, \operatorname{Pr} \cdot \operatorname{Pr}^{\prime}\right)$.

Theorem 4. The left product of two probability spaces $(\Omega, \delta, \operatorname{Pr})$ and $\left(\Omega^{\prime}, \mathcal{S}^{\prime}, \operatorname{Pr}^{\prime}\right)$ is a probability space. If $f \geqq 0$ is measurable with respect to $\mathcal{S} \cdot \mathcal{S}^{\prime}$ then

$$
\int_{\Omega \cdot \Omega^{\prime}} f\left(\omega, \omega^{\prime}\right) d \operatorname{Pr} \cdot \operatorname{Pr}^{\prime}\left(\omega, \omega^{\prime}\right)=\int_{\Omega^{\prime}}\left(\int_{\Omega} f\left(\omega, \omega^{\prime}\right) d \operatorname{Pr}(\omega)\right) d \operatorname{Pr}^{\prime}\left(\omega^{\prime}\right) .
$$

If $\left(\Omega \times \Omega^{\prime}, s \times s^{\prime}, \operatorname{Pr} \times \operatorname{Pr}^{\prime}\right)$ is the ordinary Cartesian product of the two spaces, as in [7], then $\delta \times \mathcal{S}^{\prime} \subset \mathcal{S} \cdot \mathcal{S}^{\prime}$ and $\operatorname{Pr} \cdot \operatorname{Pr}^{\prime}$ is an extension of $\operatorname{Pr} \times \operatorname{Pr}^{\prime}$.

Proof. $\mathcal{S} \cdot \mathcal{S}^{\prime}$ is closed under countable unions, since if $C_{n} \in \mathcal{S} \cdot \mathcal{S}^{\prime},\left(\cup_{n} C_{n}\right)^{\omega^{\prime}}$ $=\bigcup_{n} C_{n}^{\omega^{\prime}}$. Similarly, $\mathcal{S} \cdot \mathcal{S}^{\prime}$ is closed under complementation, since $(c C)^{\omega}$ $=\left\{\omega:\left(\omega, \omega^{\prime}\right) \notin C\right\}=c\left(C^{\omega^{\prime}}\right)$. Let $C_{n}$ be a disjoint sequence of sets in $\mathcal{S} \cdot \mathcal{S}^{\prime}$. Then

$$
\begin{aligned}
\operatorname{Pr} \cdot \operatorname{Pr}^{\prime}\left(\cup_{n} C_{n}\right) & =\int_{\Omega^{\prime}} \operatorname{Pr}\left(\cup_{n} C_{n}\right)^{\omega^{\prime}} d \operatorname{Pr}\left(\omega^{\prime}\right)=\int_{\Omega^{\prime}} \operatorname{Pr}\left(\cup_{n} C_{n}^{\omega^{\prime}}\right) d \operatorname{Pr}\left(\omega^{\prime}\right) \\
& =\int_{\Omega^{\prime}} \sum_{n} \operatorname{Pr}\left(C_{n}^{\omega^{\prime}}\right) d \operatorname{Pr}\left(\omega^{\prime}\right)=\sum_{n} \int_{\Omega^{\prime}} \operatorname{Pr}\left(C_{n}^{\omega^{\prime}}\right) d \operatorname{Pr}\left(\omega^{\prime}\right) \\
& =\sum_{n} \operatorname{Pr} \cdot \operatorname{Pr}^{\prime}\left(C_{n}\right),
\end{aligned}
$$

so that $\operatorname{Pr} \cdot \operatorname{Pr}^{\prime}$ is a countably additive measure. Clearly it is positive and $\operatorname{Pr} \cdot \operatorname{Pr}^{\prime}\left(\Omega \times \Omega^{\prime}\right)=1$.

To prove (20), note that it holds by (19) whenever $f$ is the characteristic function of a measurable set $C$ in $S \cdot S^{\prime}$. The set of $f$ for which (20) holds is closed under multiplication by positive constants and the formation of countable sums. But for any measurable $f \geqq 0$ define

$$
f_{0}\left(\omega, \omega^{\prime}\right)=\sum_{n=0}^{\infty} n \chi\left\{\left(\omega, \omega^{\prime}\right): n \leq f\left(\omega, \omega^{\prime}\right)<n+1\right\}
$$

and by recursion

$$
f_{k}\left(\omega, \omega^{\prime}\right)=2^{-k} \chi\left\{\left(\omega, \omega^{\prime}\right): 2^{-k} \leqq f\left(\omega, \omega^{\prime}\right)-\Sigma_{r-0}^{k-1} f_{r}\left(\omega, \omega^{\prime}\right)\right\} .
$$

Then $f\left(\omega, \omega^{\prime}\right)=\sum_{k=0}^{\infty} f_{k}\left(\omega, \omega^{\prime}\right)$ for all $\left(\omega, \omega^{\prime}\right)$, and so (20) hold for $f$.

If $E \in \mathcal{S}, F \in \mathcal{S}^{\prime}$ then $E \times F$ is obviously in $s \cdot \mathcal{S}^{\prime}$, so that $\delta \times \mathcal{S}^{\prime} \subset \mathcal{S} \cdot \mathcal{S}^{\prime}$. Also, $\operatorname{Pr} \cdot \operatorname{Pr}^{\prime}(E \times F)$ is by definition $\operatorname{Pr}(E) \operatorname{Pr}^{\prime}(F)=\operatorname{Pr} \times \operatorname{Pr}^{\prime}(E \times F)$, so that $\operatorname{Pr} \cdot \operatorname{Pr}^{\prime}$ is an extension of $\operatorname{Pr} \times \operatorname{Pr}^{\prime}$. This concludes the proof.

Definition. By a Markoff process (with parameter set $[0, \infty)$ ) on a measurable space $X, R$ we shall mean a family of $X$-valued measurable func- 
tions $\xi_{t}, 0 \leqq t<\infty$, on a probability space $(\Omega, \delta, \operatorname{Pr})$ (i.e., for each $F \in R$, $\left.\left\{\omega: \xi_{t}(\omega) \in F\right\} \in \mathcal{S}\right)$ such that whenever $t_{1}<\cdots<t_{n}<t$

$$
\operatorname{Pr}\left\{\xi_{t} \in F \mid \xi_{t_{1}}, \cdots, \xi_{t_{n}}\right\}(\omega)=\operatorname{Pr}\left\{\xi_{t} \in F \mid \xi_{t_{n}}\right\}(\omega) \text { a.e. }
$$

The notation in (21) for conditional probabilities is that of [4]. By a (stationary stochastic) transition function on $X$, we shall mean a function $p^{t}(x, F)$ defined for $0 \leqq t<\infty, x \in X, F \in R$ such that for each $t$ and $x, p^{t}(x, \cdot)$ is a probability measure on $X$, for each $F, p^{t}(x, F)$ is a measurable function of $(t, x)$, and such that

$$
p^{t+s}(x, F)=\int p^{t}(y, F) p^{s}(x, d y)
$$

for all $t, s, x, F$; where $p^{s}(x, d y)$ denotes the differential $d p^{s}(x, \cdot)(y)$ of the measure $p^{s}(x, \cdot)$. By a measurable function of $(t, x)$ we mean a function on $[0, \infty) \times X$ which is measurable with respect to the $\sigma$-ring $B \times A$, where $B$ consists of the Borel sets on $[0, \infty)$. We shall say that the Markoff process $\xi_{t}$ has $p^{t}(x, F)$ as its transition function in case for all $F \in R$,

$$
\operatorname{Pr}\left\{\xi_{t} \in F \mid \xi_{s}\right\}(\omega)=p^{t-s}\left(\xi_{s}(\omega), F\right) \text { a.e., }
$$$$
s<t .
$$

We shall call the probability measure $\nu$ on $X$ the initial measure in case

$$
\operatorname{Pr}\left(\left\{\omega: \xi_{0}(\omega) \in F\right\}\right)=\nu(F) .
$$

Let the $\mu^{t}, 0 \leqq t<\infty$, be a convolution semigroup of probability measures on $[0, \infty)$, as in $\S 7$. Let

$$
r^{t}(x, B)=\mu^{t}(\{y: x+y \in B\}) .
$$

Then $r^{t}(x, B)$ is a transition function on $[0, \infty)$. It follows from Kolmogoroff' theorem (see [4]) that there is a probability space $\left(\Omega^{\prime}, s^{\prime}, \operatorname{Pr}^{\prime}\right)$ and a Markoff process $\tau_{t}$ of $[0, \infty)$-valued measurable functions on $\Omega^{\prime}$ such that $\tau_{t}$ has $r^{t}(x, B)$ as its transition function and the measure $\delta, \delta(B)=\chi_{B}(0)$, as its initial measure.

Theorem 5. Let the $\xi_{t}, 0 \leqq t<\infty$, defined on the probability space $(\Omega, \mathrm{s}, \operatorname{Pr})$ be a Markoff process on the measurable space $X, \mathbb{R}$ with transition function $p^{t}(x, F)$ and initial measure $\nu$. Let $\mu^{t},\left(\Omega^{\prime}, \mathcal{s}^{\prime}, \operatorname{Pr}^{\prime}\right), \tau_{t}, \delta$ be as in the above paragraph. Then the $X$-valued functions

$$
\eta_{t}\left(\omega, \omega^{\prime}\right)=\xi_{\tau_{t}\left(\omega^{\prime}\right)}(\omega),
$$$$
0 \leqq t<\infty
$$

defined on the left product space $\left(\Omega \times \Omega^{\prime}, \mathcal{S} \cdot \mathcal{S}^{\prime}, \operatorname{Pr} \cdot \operatorname{Pr}^{\prime}\right)$ are a Markoff process with initial measure $\nu$ and transition function

$$
q^{t}(x, F)=\int_{0}^{\infty} p^{u}(x, F) d \mu^{t}(u) .
$$


Proof. For each $t, \eta_{t}$ is a measurable function with respect to $\& \cdot \mathcal{S}^{\prime}$. Fur if $F$ is in $R$ and $C=\left\{\left(\omega, \omega^{\prime}\right): \eta_{t}\left(\omega, \omega^{\prime}\right) \in F\right\}$ then for each $\omega^{\prime}, C^{\omega^{\prime}}$ $=\left\{\omega: \xi_{\tau_{t}\left(\omega^{\prime}\right)}(\omega) \in F\right\}$ is in $\delta$. Now $\phi(u)=\int p^{u}(x, F) d \nu(x)$ is a Borel-measurable function of $u$ since $p^{u}(x, F)$ is a measurable function of $(u, x)$ with respect to the $\sigma$-ring generated by products of Borel sets in $[0, \infty)$ and sets in $R$. Hence the composite function $\operatorname{Pr}\left(C^{\prime}\right)=\phi\left(\tau_{t}\left(\omega^{\prime}\right)\right)$ is a measurable function of $\omega^{\prime}$, and so $\eta_{t}$ is "left measurable"; i.e., measurable with respect to $\mathcal{S} \cdot \mathcal{S}^{\prime}$.

Let us remark that if $t \geqq s$ then

$$
\tau_{t}\left(\omega^{\prime}\right) \geqq \tau_{s}\left(\omega^{\prime}\right) \text { a.e. }
$$

since $r^{t-s}(x, \quad[0, x))=0$. Let $t_{1}<\cdots<t_{n}<t$. Define $\tau_{u}\left(\omega, \omega^{\prime}\right)=\tau_{u}\left(\omega^{\prime}\right)$, $0 \leqq u<\infty$.

Now if $F$ is in $R$, the conditional expectation

$$
\operatorname{Pr} \cdot \operatorname{Pr}^{\prime}\left\{\eta_{t} \in F \mid \eta_{t_{1}}, \cdots, \eta_{t_{n}}, \tau_{t_{1}}, \cdots, \tau_{t_{n}}\right\}\left(\omega, \omega^{\prime}\right)
$$

is equal a.e. to the conditional expectation on $\Omega^{\prime}$ :

$$
E\left\{\operatorname{Pr}\left\{\eta_{t}\left(\cdot, \omega^{\prime}\right) \in F \mid \eta_{t_{1}}\left(\cdot, \omega^{\prime}\right) \cdots, \eta_{t_{n}}\left(\cdot, \omega^{\prime}\right)\right\}(\omega) \mid \tau_{t_{1}}, \cdots, \tau_{t_{n}}\right\}\left(\omega^{\prime}\right)
$$

by $(20),=E\left\{\operatorname{Pr}\left\{\eta_{t}\left(\cdot, \omega^{\prime}\right) \in F \mid \eta_{t_{n}}\left(\cdot, \omega^{\prime}\right)\right\}(\omega) \mid \tau_{t_{1}}, \cdots, \tau_{t_{n}}\right\}\left(\omega^{\prime}\right)$ a.e. by (28), the definition (26) of $\eta_{t}$, and the Markoff property (21) of the $\xi$ 's. This in turn is equal a.e. by (23) to

$$
E\left\{p^{\tau_{t}\left(\omega^{\prime}\right)-\tau_{l_{n}}\left(\omega^{\prime}\right)}\left(\eta_{t_{n}}\left(\omega, \omega^{\prime}\right), F^{\prime}\right) \mid \tau_{l_{1}}, \cdots, \tau_{t_{n}}\right\}\left(\omega^{\prime}\right)
$$

which is equal a.e. to

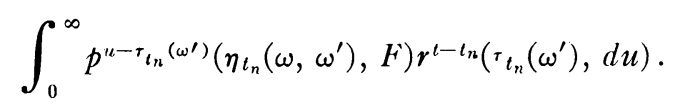

Here we have used the fact that for a Markoff process $\tau_{t}$ with transition function $r^{t}$, if $f$ is a measurable function and $t_{1}<\cdots<t_{n}<t$ then $E\left\{f\left(\tau_{t}\right) \mid \tau_{t_{1}}, \cdots, \tau_{t_{n}}\right\}\left(\omega^{\prime}\right)=\int f(u) r^{t-t_{n}}\left(\tau_{t_{n}}\left(\omega^{\prime}\right), d u\right)$ a.e. This follows from (23) applied to the $\tau_{t}$ process. In this case $f(u)=p^{u-\tau_{t_{n}}\left(\omega^{\prime}\right)}\left(\eta_{t_{n}}\left(\omega, \omega^{\prime}\right), F\right)$. Now (30) may be written as $\int_{0}^{\infty} p^{v}\left(\eta_{t_{n}}\left(\omega, \omega^{\prime}\right), F\right) d \mu^{t-t_{n}}(v)=q^{t-t_{n}}\left(\eta_{t_{n}}\left(\omega, \omega^{\prime}\right), F\right)$ by (25) and (27). That is,

$$
\operatorname{Pr} \cdot \operatorname{Pr}^{\prime}\left\{\eta_{t} \in F \mid \eta_{t_{1}}, \cdots, \eta_{t_{n}}, \tau_{t_{1}}, \cdots, \tau_{t_{n}}\right\}\left(\omega, \omega^{\prime}\right)=q^{t-t_{n}}\left(\eta_{\iota_{n}}\left(\omega, \omega^{\prime}\right), F\right)
$$

a.e. Since the right hand side is measurable with respect to the subring generated by $\eta_{t_{n}}$ alone we have a fortiori that $\operatorname{Pr} \cdot \operatorname{Pr}^{\prime}\left\{\eta_{t} \in F \mid \eta_{t_{1}}, \cdots, \eta_{t_{n}}\right\}\left(\omega, \omega^{\prime}\right)$ $=q^{t-t_{n}}\left(\eta_{t_{n}}\left(\omega, \omega^{\prime}\right), F\right)$ a.e. so that by definition (23) the $\eta_{t}$ are a Markoff process with transition function $q^{t}$. Finally, $\operatorname{Pr} \cdot \operatorname{Pr}^{\prime}\left(\left\{\left(\omega, \omega^{\prime}\right): \eta_{0}\left(\omega, \omega^{\prime}\right) \in F\right\}\right)$ $=\operatorname{Pr} \cdot \operatorname{Pr}^{\prime}\left(\left\{\left(\omega, \omega^{\prime}\right): \xi_{0}(\omega) \in F\right\}\right)$ since $\tau_{0}\left(\omega^{\prime}\right)=0$ for a.e. $\omega^{\prime}$ (the $\tau_{t}$ process has initial measure $\delta)$. But $\operatorname{Pr} \cdot \operatorname{Pr}^{\prime}\left(\left\{\left(\omega, \omega^{\prime}\right): \xi_{0}(\omega) \in F\right\}\right)=\nu(F)$, so that the Markoff process $\eta_{t}$ has initial measure $\nu$. This concludes the proof of the theorem.

As an example, consider the operator $A=d^{2} / d x^{2}$. Taken on the proper 
domain in $\mathfrak{X}=L^{p}(R), 1 \leqq p<\infty$, or $\mathfrak{X}=C(R)$, (where $R=(-\infty, \infty)$ ) this is the infinitesimal generator of the strongly continuous semigroup

$$
P^{t} f(x)=\int_{-\infty}^{\infty} \frac{1}{(4 \pi t)^{1 / 2}} e^{-(x-y)^{2} / 4 t} f(y) d y .
$$

Then $p^{t}(x, F)=P^{t} \chi_{F}(x)$ is a transition function. Let $\xi_{t}, 0 \leqq t<\infty$, defined on $(\Omega, \delta, \operatorname{Pr})$ be a Markoff process with this transition function. It is shown in [4] that the $\xi_{t}$ can be chosen so that for all $\omega$ in $\Omega, \xi_{t}(\omega)$ is a continuous function of $t$. This is called the Brownian motion or Wiener process. By Theorem 2 and the discussion of $\S 7,-\left(d^{2} / d x^{2}\right)^{\alpha}$, for $0<\alpha<1$, is the infinitesimal generator of a semigroup $Q^{t}=\int_{0}^{\infty} P^{u} d \mu^{t}(u)$ for some convolution semigroup of probability measures $\mu^{t}$ on $[0, \infty)$. The function $q^{t}(x, F)=Q^{t} \chi_{F}(x)$ is again a transition function with an associated Markoff process. These facts were discovered by S. Bochner [2]. Our results show that this is not a property peculiar to Brownian motion or the real line. Furthermore, if the $\tau_{t}$ are the random variables of a Markoff process associated with the $\mu^{t}$, then by Theorem 5 the $\xi_{r_{t}}$ are the random variables of a Markoff process with transition functions $q^{t}(x, F)$. That is, if we do not look at the sample functions $\xi_{t}(\omega)$ of Brownian motion for all values of $t$ but only at certain stochastically determined values $\tau_{t}\left(\omega^{\prime}\right)$ of the time parameter, we obtain the sample functions of the new process.

For $\alpha=1 / 2$ we obtain the transition function

$$
q^{t}(x, F)=\int_{F} \frac{1}{\pi} \frac{t}{t^{2}+(x-y)^{2}} d y .
$$

This may be verified by looking at the Fourier transforms of the two functions involved; cf. [6]. By Theorem 5, there is a Markoff process $\tau_{t}$ on $[0, \infty)$ such that the Cauchy process with transition function (32) has sample functions $\xi_{\tau_{t}}$, where the $\xi_{t}$ are the Wiener process. The fact that the Cauchy distribution in (32) and the Poisson kernel for Dirichlet's problem in a half plane are identical leads to an interesting interpretation of the $\tau_{t}$. They are the first passage times of the Wiener process.

Theorem 6. Let $\xi_{t}$ on $(\Omega, s, \operatorname{Pr})$ and $\xi_{t}^{\prime}$ on $\left(\Omega^{\prime}, \mathcal{s}^{\prime}, \operatorname{Pr}^{\prime}\right), 0 \leqq t<\infty$, both be Wiener processes with initial measure $\delta$ (mass 1 concentrated at 0 ). Let the $\xi_{t}^{\prime}\left(\omega^{\prime}\right)$ be such that for all $\omega^{\prime}, \xi_{t}^{\prime}\left(\omega^{\prime}\right)$ is a continuous function of $t$ (as in [4]). Let

$$
\tau_{t}\left(\omega^{\prime}\right)=\inf \left\{s: \xi_{s}^{\prime}\left(\omega^{\prime}\right)=t\right\} .
$$

Then the $\xi_{\tau_{t}\left(\omega^{\prime}\right)}(\omega)$ on $\left(\Omega \times \Omega^{\prime}, s \cdot \mathcal{S}^{\prime}, \operatorname{Pr} \cdot \operatorname{Pr}^{\prime}\right)$ are the Cauchy process with transition function (32).

Proof. Let

$$
\mu^{t}(B)=\operatorname{Pr}^{\prime}\left(\left\{\omega^{\prime}: \tau_{t}\left(\omega^{\prime}\right) \in B\right\}\right)
$$


for all Borel sets $B$ in $[0, \infty)$. Then each $\mu^{t}$ is a probability measure on $[0, \infty)$. That they constitute a convolution semigroup follows immediately from the fact that $\operatorname{Pr}^{\prime}\left(\left\{\omega^{\prime}: \tau_{t}\left(\omega^{\prime}\right)+\tau_{s}\left(\omega^{\prime}\right) \in B\right\}\right)=\operatorname{Pr}^{\prime}\left(\left\{\omega^{\prime}: \tau_{t+s}\left(\omega^{\prime}\right) \in B\right\}\right)$. By Theorem 5 , the $\xi_{\tau_{t}\left(\omega^{\prime}\right)}(\omega)$ on $\Omega \cdot \Omega^{\prime}$ are a Markoff process with some transition function $q^{t}$.

Let $f$ be a bounded continuous function on $R$. Then $f\left(\xi_{\tau_{t}\left(\omega^{\prime}\right)}(\omega)\right)$ is the value of $f\left(\xi_{u}(\omega)\right)$ for the least value of $u$ for which $\xi_{u}^{\prime}\left(\omega^{\prime}\right)=t$. Therefore by a theorem of Doob [5, Theorem 6.2], $E\left\{f\left(\xi_{\tau_{t+h}}\right) \mid \xi_{h}\right\}(\omega)$ is a harmonic function of $\left(t, \xi_{h}(\omega)\right)$ and is given by convolution with the Poisson kernel. That is,

$$
\int_{-\infty}^{\infty} f(y) q^{t}\left(\xi_{h}(\omega), d y\right)=E\left\{f\left(\xi_{\tau_{t}}\right) \mid \xi_{h}\right\}(\omega)=\int_{-\infty}^{\infty} f(y) \frac{1}{\pi} \frac{t}{t^{2}+\left(\xi_{h}(\omega)-y\right)^{2}} d y \text { a.e. }
$$

Since this holds for all bounded continuous functions $f$ and since $\xi_{h}(\omega)$ may take a.e. value of $x$, it follows that $q^{t}(x, F)$ is given by (32) for a.e. $x$, and the theorem follows.

CorollaRy. If the $\xi_{t}$ defined on $(\Omega, s, \operatorname{Pr})$ are an arbitrary Markoff process on $X$ with transition function $p^{t}(x, F)$, if the $\xi_{t}^{\prime}$ defined on $\left(\Omega^{\prime}, \mathrm{s}^{\prime}, \mathrm{Pr}^{\prime}\right)$ are the Wiener process and $\tau_{t}$ is defined by (33), then the $\xi_{\tau_{t}\left(\omega^{\prime}\right)}(\omega)$ on $\Omega \cdot \Omega^{\prime}$ are the sample functions of a Markoff process on $X$ with transition function

$$
q^{t}(x, F)=\int_{0}^{\infty} p^{u}(x, F) d \mu^{t}(u)
$$

where $\mu^{t}$ is defined by (34). If on some Banach space $\mathfrak{X}$ of functions, $P^{t} f(x)$ $=\int f(y) p^{t}(x, d y)$ constitutes a strongly continuous semigroup with infinitesimal generator $A$ then $Q^{\prime} f(x)=\int f(y) q^{t}(x, d y)$ is a strongly continuous semigroup with infinitesimal generator $-(-A)^{1 / 2}$, and

$$
-(-A)^{1 / 2} f=\frac{1}{2(\pi)^{1 / 2}} \int_{0}^{\infty} \frac{P^{t} f-f}{t^{3 / 2}} d t \text { for all } f \in D(A) .
$$

Proof. The first statement follows from Theorem 5 as in the first paragraph of the proof of Theorem 6 . The second statement holds for the case that $\xi_{t}$ is the Wiener process, by Theorem 5 (the formula (35) holds by Theofem (2)). By the discussion in $\$ 7$, this means that $\tilde{\mu}^{t}(\lambda)=e^{-(-\lambda)^{\alpha} t}$ for $\alpha=1 / 2$ and so (35) holds in the general case.

Let us restate Theorem 6 more informally. We have two independent Brownian motions $\xi$ and $\xi^{\prime}$. For all $t$, we wait until the $\xi^{\prime}$ particle first reaches $t$ and simultaneously observe the $\xi$ particle. Then the $\xi$ particle appears to move according to the Cauchy law.

\section{REFERENCES}

1. A. V. Balakrishnan, An operational calculus for closed operators, Bull. Amer. Math. Soc. Abstract 61-2-220. 
2. S. Bochner, Diffusion equation and stochastic processes, Proc. Nat. Acad. Sci. USA vol. 35 (1949) pp. 368-370.

3. - Harmonic analysis and the theory of probability, Berkeley, 1955.

4. J. L. Doob, Stochastic processes, New York, John Wiley \& Sons, 1953.

5. - Semimartingales and subharmonic functions, Trans. Amer. Math. Soc. vol. 77 (1954) pp. 86-121.

6. W. Feller, On a generalization of Marcel Riesz' potentials and the semi-groups generated by them, Communications du séminaire mathématique de l'université de Lund, tome supplémentaire, 1952, dédié à Marcel Riesz.

7. P. R. Halmos, Measure theory, New York, Van Nostrand, 1950.

8. Einar Hille, Functional analysis and semigroups, Amer. Math. Soc. Colloquium Pub. lications, vol. 31, New York, 1948.

9. L. H. Loomis, An introduction to abstract harmonic analysis, New York, Van Nostrand, 1953.

10. R. S. Phillips, On the generation of semigroups of linear operators, Pacific J. Math. vol. 2 (1952) pp. 343-369.

11. Laurent Schwartz, Théorie des distributions, vols. 1 and 2, Paris, Hermann et Cie, 1951.

Institute for Advanced Study,

Princeton, N. J. 